\title{
Erratum to: Principles of a Prototype Model of a Device for Automatic Control of Cardio-Pulmonary Resuscitation Procedures
}

Artem Bureev, Ekaterina Dikman, Ivan Zemlyakov, Artem Osipov, Anton Seleznev, and Liudmila Khohlova National Research Tomsk State University, 634050, Tomsk, Russia

Original article:

MATEC Web of Conferences 79, 01011 (2016), DOI: 10.1051/matecconf/20167901011

In the section Acknowledgement, the line “, and the program of improving competitiveness of the TSU (project No. 8.2.31.2015)" must be deleted. 